\section{Vol. 31, Issue 6, June 2008}

Editorials

297 Brain Metastases in Very Young Patients with Lung Cancer Are Still Brain Metastases

Gore, E.M. (Milwaukee, WI)

300 Management of Malignant Glioma - Quo Vadis? Stupp, R. (Lausanne); Hottinger, A.F. (Geneva)

Original Articles

305 Disease Presentation and Treatment Outcome in Very Young Patients with Brain Metastases from Lung Cancer

Nieder, C. (Bodø); Thamm, R.; Astner, S.T.; Molls, M. (München)

309 Temozolomide in Newly Diagnosed Malignant Gliomas: Administered Concomitantly with Radiotherapy, and Thereafter as Consolidation Treatment

Yaman, E.; Buyukberber, S.; Uner, A.; Coskun, U.; Akmansu, M.; Benekli, M.; Yamac, D.; Ozturk, B.; Kaya, A.O.; Yildiz, R.; Ozkan, S.; Gunel, N. (Ankara)

315 Serum CA-125 Level After 6 Cycles of Primary Adjuvant Chemotherapy Is a Useful Prognostic Factor for Complete Responders' Survival in Patients with Advanced Epithelial Ovarian Cancer Kim, H.S.; Park, N.H.; Chung, H.H.; Kim, J.W.; Song, Y.S.; Kang, S.B. (Seoul)

\section{Clinical Cases}

321 Six Cases of Chylous Leakage after Axillary Lymph Node Dissection

Cong, M.H.; Liu, Q.; Zhou, W.H.; Zhu, J.; Song, C.X.; Tian, X.S. (Shandong)

325 Repeated and Preemptive Palliative Radiotherapy of Symptomatic Hepatomegaly in a Patient with Advanced Myelofibrosis

Riesterer, O.; Gmür, J.; Lütolf, U. (Zürich)

\section{Band 31, Heft 6, Juni 2008}

Editorials

297 Hirnmetastasen bei sehr jungen Lungenkrebspatienten sind immer noch Hirnmetastasen Gore, E.M. (Milwaukee, WI)

300 Behandlung des malignen Glioms - quo vadis? Stupp, R. (Lausanne); Hottinger, A.F. (Geneva)

Originalarbeiten

305 Krankheitsausmaß und Behandlungsergebnisse bei sehr jungen Patienten mit Hirnmetastasen eines Bronchialkarzinoms Nieder, C. (Bodø); Thamm, R.; Astner, S.T.; Molls, M. (München)

309 Temozolomid bei neu diagnostiziertem malignen Gliom: Simultan zur Bestrahlung und anschließend als Konsolidierungstherapie

Yaman, E.; Buyukberber, S.; Uner, A.; Coskun, U.; Akmansu, M.; Benekli, M.; Yamac, D.; Ozturk, B.; Kaya, A.O.; Yildiz, R.; Ozkan, S.; Gunel, N. (Ankara)

315 Der CA-125-Serumspiegel nach 6 Zyklen einer primären adjuvanten Chemotherapie ist ein nuitzlicher Prognosefaktor für das Überleben von Patienten mit fortgeschrittenem epithelialem Ovarialkarzinom, die ein komplettes Ansprechen zeigen Kim, H.S.; Park, N.H.; Chung, H.H.; Kim, J.W.; Song, Y.S.; Kang, S.B. (Seoul)

\section{Kasuistiken}

321 Sechs Fälle von Chylusaustritt nach axillärer Lymphknotenentfernung Cong, M.H.; Liu, Q.; Zhou, W.H.; Zhu, J.; Song, C.X.; Tian, X.S. (Shandong)

325 Wiederholte und präemptive palliative Radiotherapie einer symptomatischen Hepatomegalie bei einem Patienten mit fortgeschrittener Osteomyelofibrose Riesterer, O.; Gmür, J.; Lütolf, U. (Zürich)

\section{KARGER}

Fax +497614520714

E-mail Information@Karger.de

www.karger.com 


\section{Vol. 31, Issue 6, June 2008}

328 Extensive Rendu-Osler Disease in a Breast Cancer

Patient: Increasing Hepatic Arteriovenous

Malformations under Endocrine Therapy

Mimicking Liver Metastases

Battista, M.J.; Eichbaum, M.H.R.; Hosch, W.P.; Fersis, N.;

Sohn, C. (Heidelberg)

332 Extensive Genital and Gluteal Epitheliolyses

Caused by Locoregional Lichen Ruber Two Years

after Primary Treatment of Vulvar Cancer

Eichbaum, M.; Harms, W.; Bolz, S.; Gebauer, G.; Schneeweiss, A.;

Sohn, C. (Heidelberg)

Review Article

335 Modern Treatment Options for Elderly Patients with Multiple Myeloma

Görner, M. (Bielefeld); Späth-Schwalbe, E. (Berlin) for the Task Force Geriatrische Onkologie of the DGHO, ÖGHO, DGG and AIO

Letter to the Editors

343 Longitudinal Analysis of Psychiatric Morbidity

in Cancer Patients

Bringmann, H.; Singer, S.; Höckel, M.; Stolzenburg, J.U.; Krauss, O.; Schwarz, R. (Leipzig)

346 PharmaNews / PharmaTicker

349 Meetings and Conferences

324 Imprint

351 Guidelines for Authors

Forthcoming papers are listed on page 352.

\section{Band 31, Heft 6, Juni 2008}

328 Ausgedehnter M. Rendu-Osler bei einer Mammakarzinompatientin: Größenprogrediente hepatische, arteriovenöse Malformationen unter endokriner Therapie imitieren Lebermetastasen Battista, M.J.; Eichbaum, M.H.R.; Hosch, W.P.; Fersis, N.; Sohn, C. (Heidelberg)

332 Extensive genitale und gluteale Epitheliolysen durch einen locoregionären Lichen ruber zwei Jahre nach Primärtherapie eines Vulvakarzinoms Eichbaum, M.; Harms, W.; Bolz, S.; Gebauer, G.; Schneeweiss, A.; Sohn, C. (Heidelberg)

\section{Übersichtsarbeit}

335 Moderne Therapieoptionen für ältere Patienten mit Multiplem Myelom

Görner, M. (Bielefeld); Späth-Schwalbe, E. (Berlin) for the Task Force Geriatrische Onkologie of the DGHO, ÖGHO, DGG and AIO

Brief an die Herausgeber

343 Longitudinalanalyse psychischer Erkrankungen bei Krebspatienten

Bringmann, H.; Singer, S.; Höckel, M.; Stolzenburg, J.U.; Krauss, O.; Schwarz, R. (Leipzig)

346 PharmaNews / PharmaTicker

349 Tagungen und Kongresse

324 Impressum

351 Hinweise für Autoren

Einen Ausblick auf den Inhalt der kommenden Hefte finden Sie auf Seite 352.

\section{KARGER}

Fax +497614520714

E-mail Information@Karger.de

www.karger.com
(C) 2008 S. Karger GmbH, Freiburg 